\title{
P69 The Initial Upstroke Time is Most Strongly Associated with the Severity of as Among Brachial Pulse Wave Parameters
} \author{
Tetsuya Ito $^{2}$, Kazuomi Kario ${ }^{3}$ \\ ${ }^{1}$ Department of Cardiovascular Medicine, Jichi Medical University, Shimotsuke, Japan \\ ${ }^{2}$ Academic Promotion Section, Fukuda Denshi Co., Ltd. \\ ${ }^{3}$ Jichi Medical University, Japan \\ ${ }^{4}$ ReD Head Office, Fukuda Denshi Co., Ltd.
}

Masao Takahashi ${ }^{1, *}$, Kyohei Fukatani ${ }^{2}$, Tomoyuki Kabutoya ${ }^{3}$, Satoshi Hoshide ${ }^{3}$, Tatsuya Yoneyama ${ }^{4}$,

\begin{abstract}
Background: Aortic valve stenosis (AS) is the most common valve disease in an elderly population, therefore, simple screening examination for AS is needed. Although a prolonged carotid upstroke time (UT), and prolonged ejection time (ET) of a brachial pulse wave (BPW) have been observed in severe AS patients, it has been unclear which BPW parameters have a better correlation with the severity of AS. The aim of this study was to examine which BPW parameters are most relevant to the severity of AS.

Methods: Sixty-five Consecutive moderate and severe AS patients who were evaluated by trans-thoracic echocardiography were enrolled in this study. Control patients who were adjusted for age, gender, and blood pressure among outpatients were enrolled $(N=110)$. UT, ET, initial upstroke time (iUT), and half rise time of upstroke $(1 / 2 \mathrm{hrUT})$ were evaluated correlations between mean pressure gradient ( $\mathrm{mPG}$ ) among AS patients.

Results: iUT and $1 / 2$ hrUT have significant correlations with $\mathrm{mPG}$ among AS patients (iUT: $R=0.50,95 \% \mathrm{CI}=0.29-0.67$, $p<0.0001$; $1 / 2$ hrUT: $R=0.41,95 \% \mathrm{CI}=0.19-0.60, p<0.001)$, whereas UT and ET did not. Multivariate logistic regression analysis showed area under curve (AUC) of iUT and $1 / 2 \mathrm{hrUT}$ were higher than UT and ET to predict $\mathrm{mPG}>40 \mathrm{mmHg}$ (AUC: iUT vs $1 / 2$ hrUT vs UT vs ET $=0.90$ vs 0.89 vs 0.69 vs 0.77 ).

Conclusion: The severity of AS appeared strongly in the first half of the BPW upstroke. iUT and 1/2 hrUT may be a simple and useful screening test to assess the severity of AS.

(c) 2019 Association for Research into Arterial Structure and Physiology. Publishing services by Atlantis Press International B.V. This is an open access article distributed under the CC BY-NC 4.0 license (http://creativecommons.org/licenses/by-nc/4.0/).
\end{abstract}

